\title{
External area integral inequality for the Cauchy-Leray-Fantappiè integral
}

\author{
Alexander Rotkevich ${ }^{\mathrm{a}}$ \\ ${ }^{a}$ Department of Mathematical analysis, Mathematics and Mechanics Faculty, St. Petersburg \\ State University, 198504, Universitetsky prospekt, 28, Peterhof, St. Petersburg, Russia
}

\begin{abstract}
In this paper we extend Luzin inequality for functions defined by the CauchyLeray-Fantappiè integral on the complement of a convex domain in $\mathbb{C}^{n}$.

Keywords: Area inequality, Cauchy-Leray-Fantappiè integral

2010 MSC: 32E30, 41A10
\end{abstract}

\section{Introduction}

Let $G \subset \mathbb{C}$ be a Radon domain, for $z \in \partial G$ we consider a sector $S(z)=\{\xi \in$ $\left.G: \operatorname{dist}((, \xi), \partial G) \geq \frac{1}{2}|\xi-z|\right\}$. It is well known (see [18], [3]), that for function $f$ holomorphic on $G$ one has

$$
\left\|I_{f}\right\|_{L^{p}(\partial G)} \leq c(p, G)\|f\|_{L^{p}(\partial G)}, 1<p<\infty
$$

with some constant $c(p, G)$, where $I_{f}$ is an area-integral

$$
I_{f}(z)=\left(\int_{S(z)}\left|f^{\prime}(\xi)\right|^{2} d \mu(\xi)\right)^{1 / 2}
$$

and $d \mu$ is Lebesgue measure on $\mathbb{C}$.

There are many many generalizations of this inequality for holomorphic functions on regular domains in $\mathbb{C}^{n}$ by P. Ahern, J. Bruna ([1]), A. Nagel, E.M. Stein, S. Wainger [11], G. Sardine [15], S. Krantz and S.Y. Lee [6]. Our main

Email address: rotkevichas@gmail.com (Alexander Rotkevich)

The work is supported by Russian Science Foundation Grant 14-41-00010.

Preprint submitted to Elsevier

September 24, 2018 
result (theorems 4.1, 4.2) is extension of this inequality to functions generated by Cauchy-Leray-Fantappiè integral and defined on the complement of a convex domain in $\mathbb{C}^{n}$. The motivation of this paper is the possibility of applications of these results to the characterization of spaces of analytic functions by pseudoanalytical extensions (see [14]).

\section{Main notations and definitions}

Let $\mathbb{C}^{n}$ be the space of $n$ complex variables, $n \geq 2, z=\left(z_{1}, \ldots, z_{n}\right), z_{j}=$ $x_{j}+i y_{j}$

$$
\begin{gathered}
\partial_{j} f=\frac{\partial f}{\partial z_{j}}=\frac{1}{2}\left(\frac{\partial f}{\partial x_{j}}-i \frac{\partial f}{\partial y_{j}}\right), \quad \bar{\partial}_{j} f=\frac{\partial f}{\partial \bar{z}_{j}}=\frac{1}{2}\left(\frac{\partial f}{\partial x_{j}}+i \frac{\partial f}{\partial y_{j}}\right), \\
\partial f=\sum_{k=1}^{n} \frac{\partial f}{\partial z_{k}} d z_{k}, \quad \bar{\partial} f=\sum_{k=1}^{n} \frac{\partial f}{\partial \bar{z}_{k}} d \bar{z}_{k}, \quad d f=\partial f+\bar{\partial} f .
\end{gathered}
$$

The notation

$$
\langle\partial f(z), w\rangle=\sum_{k=1}^{n} \frac{\partial f(z)}{\partial z_{k}} w_{k} .
$$

is used to indicate the action of $\partial f$ on the vector $w \in \mathbb{C}^{n}$, and

$$
|\bar{\partial} f|=\left|\frac{\partial f}{\partial z_{1}}\right|+\ldots+\left|\frac{\partial f}{\partial z_{n}}\right| .
$$

The euclidean distance form the point $z \in \mathbb{C}^{n}$ to the set $D \subset \mathbb{C}^{n}$ we denote as $\operatorname{dist}(z, D)=\inf \{|z-w|: w \in D\}$. Lebesgue measure in $\mathbb{C}^{n}$ we denote as $d \mu$.

For a multiindex $\alpha=\left(\alpha_{1}, \ldots, \alpha_{n}\right) \in \mathbb{N}^{n}$ we set $|\alpha|=\alpha_{1}+\ldots+\alpha_{n}$ and $\alpha !=\alpha_{1} ! \ldots \alpha_{2} !$, also $z^{\alpha}=z_{1}^{\alpha_{1}} \ldots z_{n}^{\alpha_{n}}$ and $\partial^{\alpha} f=\frac{\partial^{|\alpha|} f}{\partial \bar{z}_{1}^{\alpha_{1}} \ldots \partial \bar{z}_{n}^{\alpha_{n}}}$.

Let $\Omega=\left\{z \in \mathbb{C}^{n}: \rho(z)<0\right\}$ be a strongly convex domain with a $C^{3}$-smooth defining function. We need to consider a family of domains

$$
\Omega_{t}=\left\{z \in \mathbb{C}^{n}: \rho(z)<t\right\}
$$

that are also strongly convex for each $|t|<\varepsilon$, where $\varepsilon>0$ is small enough, that is $d^{2} \rho(z)$ is positive definite when $|\rho(z)| \leq \varepsilon$. For $z \in \Omega_{\varepsilon} \backslash \Omega_{-\varepsilon}$ we denote the nearest point on $\partial \Omega$ as $\operatorname{pr}_{\partial \Omega}(z)$. Then the mapping

$$
\operatorname{pr}_{\partial \Omega}: \Omega_{\varepsilon} \backslash \Omega_{-\varepsilon} \rightarrow \partial \Omega
$$


is well defined, $C^{2}-$ smooth on $\Omega_{\varepsilon} \backslash \Omega$ and $\left|z-\operatorname{pr}_{\partial \Omega}(z)\right|=\operatorname{dist}(z, \partial \Omega)$.

For $\xi \in \partial \Omega_{t}$ we define the complex tangent space

$$
T_{\xi}=\left\{z \in \mathbb{C}^{n}:\langle\partial \rho(\xi), \xi-z\rangle=0\right\}
$$

The space of holomorphic functions we denote as $H(\Omega)$. Throughout this paper we use notations $\lesssim, \asymp$. We let $f \lesssim g$ if $f \leq c g$ for some constant $c>0$, that doesn't depend on main arguments of functions $f$ and $g$ and usually depend only on dimension $n$ and domain $\Omega$. Also $f \asymp g$ if $c^{-1} g \leq f \leq c g$ for some $c>1$.

\section{Cauchy-Leray-Fantappiè formula}

In the context of theory of several complex variables there is no unique reproducing formula formula, however we could use the Leray theorem, that allows us to construct holomorphic reproducing kernels ([2], [10], [12]). For convex domain $\Omega=\left\{z \in \mathbb{C}^{n}: \rho(z)<0\right\}$ this theorem brings us Cauchy-LerayFantappiè formula, and for $f \in H^{1}(\Omega)$ and $z \in \Omega$ we have

$$
f(z)=K_{\Omega} f(z)=\frac{1}{(2 \pi i)^{n}} \int_{\partial \Omega} \frac{f(\xi) \partial \rho(\xi) \wedge(\bar{\partial} \partial \rho(\xi))^{n-1}}{\langle\partial \rho(\xi), \xi-z\rangle^{n}}=\int_{\partial \Omega} f(\xi) K(\xi, z) \omega(\xi),
$$

where $\omega(\xi)=\frac{1}{(2 \pi i)^{n}} \partial \rho(\xi) \wedge(\bar{\partial} \partial \rho(\xi))^{n-1}$, and $K(\xi, z)=\langle\partial \rho(\xi), \xi-z\rangle^{-n}$.

The $(2 n-1)$-form $\omega$ defines on $\partial \Omega_{t}$ Leray-Levy measure $d S$ that is equivalent to Lebesgue surface measure $d \sigma_{t}$ (for details see [2], [8], [9]). This allows us to identify Lebesgue spaces defined with respect to measures $d \sigma_{t}$ and $d S$. Also note, that measure $d V$ defined by the $2 n$-form $d \omega=(\partial \bar{\partial} \rho)^{n}$ is equivalent to Lebesgue measure $d \mu$ in $\mathbb{C}^{n}$.

By [13] the integral operator $K_{\Omega}$ defines a bounded mapping on $L^{p}(\partial \Omega)$ to $H^{p}(\Omega)$ for $1<p<\infty$.

The function $d(w, z)=|\langle\partial \rho(w), w-z\rangle|$ defines on $\partial \Omega$ quasimetric, and if $B(z, \delta)=\{w \in \partial \Omega: d(w, z)<\delta\}$ is a quasiball with respect to $d$ then $\sigma(B(z, \delta)) \asymp \delta^{n}$, see for example [13]. Therefore $\{\partial \Omega, d, \sigma\}$ is a space of homogeneous type. 
Note also the crucial role in the forthcoming considerations of the following estimate that is proved in [14].

Lemma 3.1. Let $\Omega$ be strongly convex, then

$$
d(w, z) \asymp \rho(w)+d\left(\operatorname{pr}_{\partial \Omega}(w), z\right), w \in \mathbb{C}^{n} \backslash \Omega, z \in \partial \Omega .
$$

\subsection{Korányi regions}

For $\xi \in \partial \Omega$ and $\varepsilon>0$ we define the inner Korányi region as

$$
D^{i}(\xi, \eta, \varepsilon)=\left\{\tau \in \Omega: \operatorname{pr}_{\partial \Omega}(\tau) \in B(\xi,-\eta \rho(\tau)), \rho(\tau)>-\varepsilon\right\} .
$$

The strong convexity of $\Omega$ implies that area-integral inequality by S. Krantz and S.Y. Li [6] for $f \in H^{p}(\Omega), 0<p<\infty$, could be expressed as

$$
\int_{\partial \Omega} d \sigma(z)\left(\int_{D^{i}(z, \eta, \varepsilon)}|\partial f(\tau)|^{2} \frac{d \mu(\tau)}{(-\rho(\tau))^{n-1}}\right)^{p / 2} \leq c(\Omega, p) \int_{\partial \Omega}|f|^{p} d \sigma .
$$

Consider the decomposition of vector $\tau \in \mathbb{C}^{n}$ as $\tau=w+\operatorname{tn}(\xi)$, where $w \in T_{\xi}, t \in \mathbb{C}$, and $n(\xi)=\frac{\bar{\partial} \rho(\xi)}{|\bar{\partial} \rho(\xi)|}$ is a complex normal vector at $\xi$. We define the external Korányi region as

$$
\begin{aligned}
D^{e}(\xi, \eta, \varepsilon)= & \left\{\tau \in \mathbb{C}^{n} \backslash \Omega: \tau=w+\operatorname{tn}(\xi),\right. \\
& \left.w \in T_{\xi}, t \in \mathbb{C},|w|<\sqrt{\eta \rho(\tau)},|\operatorname{Im}(t)|<\eta \rho(\tau), \rho(\tau)<\varepsilon\right\} .
\end{aligned}
$$

The main result of this paper is the area-integral inequality similar to (4) for external regions $D^{e}(\xi, \eta, \varepsilon)$.

We point out two rules for integration over regions $D^{e}(\xi, \eta, \varepsilon)$. First, for every function $F$ we have

$$
\int_{\Omega_{\varepsilon} \backslash \Omega}|F(z)| d \mu(z) \asymp \int_{\partial \Omega} d \sigma(\xi) \int_{D^{e}(\xi, \eta, \varepsilon)}|F(\tau)| \frac{d \mu(\tau)}{\rho(\tau)^{n}} .
$$

Second, if $F(w)=\tilde{F}(\rho(w))$ then

$$
\int_{D^{e}(\xi, \eta, \varepsilon)}|F(\tau)| d \mu(\tau) \asymp \int_{0}^{\varepsilon}|\tilde{F}(t)| t^{n} d t .
$$


Similar rules are valid for regions $D^{i}(\xi, \eta, \varepsilon)$.

We could clarify the estimate of $d(\tau, w)$ in lemma 3.1 for $\tau \in D^{e}(z, \eta, \varepsilon)$.

Lemma 3.2. Let $\Omega$ be a strongly convex domain and $\varepsilon, \eta>0$, then

$$
d(\tau, w) \asymp \rho(\tau)+d(z, w), \quad z, w \in \partial \Omega, \tau \in D^{e}(z, \eta, \varepsilon) .
$$

Proof. For $\tau \in D^{e}(z, \eta, \varepsilon)$ we denote $\hat{\tau}=\operatorname{pr}_{\partial \Omega}(\tau)$, then $d(\hat{\tau}, z) \lesssim \eta \rho(\tau)$ and by lemma 3.1

$$
d(\tau, w) \lesssim \rho(\tau)+d(\hat{\tau}, w) \lesssim \rho(\tau)+d(\hat{\tau}, z)+d(z, w) \lesssim \rho(\tau)+d(z, w)
$$

On the other hand,

$$
\begin{aligned}
\rho(\tau)+d(z, w) \lesssim \rho(\tau)+(d(z, \hat{\tau})+d(\hat{\tau}, w)) \lesssim(1 & +\eta) \rho(\tau)+d(\hat{\tau}, w) \\
& \lesssim \rho(\tau)+d(\hat{\tau}, w) \lesssim d(\tau, w) .
\end{aligned}
$$

\section{Area-integral inequality for external Korányi region}

Let $\Omega \subset \mathbb{C}^{n}$ be a strongly convex domain and $\eta, \varepsilon>0$. For function $g \in$ $L^{1}(\partial \Omega)$ and $l \in \mathbb{N}$ we define a function

$$
I_{l}(g, z)=\left(\int_{D^{e}(z, \eta, \varepsilon)}\left|\int_{\partial \Omega} \frac{g(w) d S(w)}{\langle\partial \rho(\tau), \tau-w\rangle^{n+l}}\right|^{2} d \nu_{l}(\tau)\right)^{1 / 2}
$$

where $d S(w)=\frac{1}{(2 \pi i)^{n}} \partial \rho(w) \wedge(\bar{\partial} \partial \rho(w))^{n-1}($ see $(3))$ and $d \nu_{l}(\tau)=\frac{d \mu(\tau)}{\rho(\tau)^{n-2 l-1}}$.

Theorem 4.1. Let $\Omega$ be strongly convex domain and $g \in L^{p}(\partial \Omega), 1<p<\infty$, Then

$$
\int_{\partial \Omega} I_{l}(g, z)^{p} d \sigma(z) \lesssim \int_{\partial \Omega}|g(z)|^{p} d \sigma(z) .
$$

Note that in the one-variable case the integral (6) is a holomorphic function and the result of the theorem follows from [3]. 
Recall that (semi)norm in $\mathrm{BMO}=\mathrm{BMO}(\partial \Omega)$ is defined by

$$
\|f\|=\sup \frac{1}{\sigma(B)} \int_{B}\left|f-f_{B}\right| d \sigma,
$$

where $f_{B}=\sup \frac{1}{\sigma(B)} \int_{B} f d \sigma$ is the average value of $f$ on the quasiball $B$ and the supremum is taken over all quasiballs $B \subset \partial \Omega$. We prove that operator $I_{l}$ is also bounded on BMO.

Theorem 4.2. Let $\Omega$ be strongly convex domain then

$$
\left\|I_{l}(g)\right\|_{\mathrm{BMO}} \lesssim\|g\|_{\mathrm{BMO}}
$$

The main idea of proof of these theorems is that the operator $I_{l}$ could be considered as a sum of operators with values in some model $L^{2}$-space on function (see decomposition (15) and formula (16) for kernel).

Definition 4.1. Assume, that defining function $\rho$ for strongly convex domain $\Omega$ has the following form near $0 \in \partial \Omega$

$$
\rho(z)=2 \operatorname{Re}\left(z_{n}\right)+\sum_{j, k=1}^{n} A_{j k} z_{j} \bar{z}_{k}+O\left(|z|^{3}\right)
$$

with positive definite form $A_{j k} z_{j} \bar{z}_{k}$. We define a set

$$
\begin{aligned}
D_{0}(\eta, \varepsilon)=\left\{\tau \in \mathbb{C}^{n} \backslash \Omega:\left|\tau_{1}\right|^{2}+\ldots+\left|\tau_{n-1}\right|^{2}<\eta \operatorname{Re}\left(\tau_{n}\right),\right. \\
\left.\left|\operatorname{Im}\left(\tau_{n}\right)\right|<\eta \operatorname{Re}\left(\tau_{n}\right),\left|\operatorname{Re}\left(\tau_{n}\right)\right|<\varepsilon\right\} .
\end{aligned}
$$

Lemma 4.3. Suppose, that $\rho$ has the form (9). There exist constants $c, \varepsilon_{0}>0$ such that

$$
D^{e}(0, \eta, \varepsilon) \subset D_{0}(c \eta, c \varepsilon), D_{0}(\eta, \varepsilon) \subset D^{e}(0, c \eta, c \varepsilon) \text { for } 0<\eta, \varepsilon<\varepsilon_{0} .
$$

Proof. For the function $\rho$ of the form (9) the Korányi sector (5) could be expressed as follows

$$
\begin{aligned}
D^{e}(0, \eta, \varepsilon)=\left\{\tau \in \mathbb{C}^{n} \backslash \Omega:\left|\tau_{1}\right|^{2}+\ldots+\left|\tau_{n-1}\right|^{2}\right. & \leq \eta \rho(\tau), \\
& \left.\left|\operatorname{Im}\left(\tau_{n}\right)\right| \leq \eta \rho(\tau), \rho(\tau)<\varepsilon\right\}
\end{aligned}
$$


and

$$
\begin{aligned}
& \rho(\tau) \leq 2 \operatorname{Re}\left(\tau_{n}\right)+c_{0}\left(\left|\tau_{1}\right|^{2}+\ldots+\left|\tau_{n-1}\right|^{2}+\operatorname{Im}\left(\tau_{n}\right)^{2}+\operatorname{Re}\left(\tau_{n}\right)^{2}\right) \\
& \leq\left(2+c_{0} \operatorname{Re}\left(\tau_{n}\right)\right) \operatorname{Re}\left(\tau_{n}\right)+c_{0}(1+\eta \rho(\tau)) \eta \rho(\tau), \tau \in D^{e}(0, \eta, \varepsilon) .
\end{aligned}
$$

Thus for $\eta<\eta_{0}=\frac{1}{8 c_{0}}$ we have $\rho(\tau) \leq c \operatorname{Re}\left(\tau_{n}\right)$.

It is easy to see, that $|\tau| \rightarrow 0$ when $\rho(\tau) \rightarrow 0, \tau \in D^{e}(0, \eta, \varepsilon)$. Then by convexity of $\Omega$

$$
2 \operatorname{Re}\left(\tau_{n}\right)=\rho(\tau)-\sum_{j, k=1}^{n} A_{j k} \tau_{j} \bar{\tau}_{k}+O\left(|\tau|^{3}\right) \leq \rho(\tau), \tau \in D^{e}\left(0, \eta, \varepsilon_{0}\right)
$$

for some $\varepsilon_{0} \in\left(0, \eta_{0}\right)$.

Finally $D^{e}(0, \eta, \varepsilon) \subset D_{0}(c \eta, \varepsilon)$ and analogously $D_{0}(\eta, \varepsilon) \subset D^{e}(0, \eta, \varepsilon)$ for $0<\eta, \varepsilon<\varepsilon_{0}$.

Theorem 4.4. There exists such covering of the set $\bar{\Omega}_{\varepsilon} \backslash \Omega_{-\varepsilon}$ by open sets $\Gamma_{j}$ such that for every $\xi \in \Gamma_{j}$ we can find a holomorphic change of coordinates $\varphi_{j}(\xi, \cdot): \mathbb{C}^{n} \rightarrow \mathbb{C}^{n}$ such that

1. The mapping $\varphi_{j}(\xi, \cdot)$ transforms function $\rho$ to the type (9) and could be expressed as follows

$$
\varphi_{j}(\xi, z)=\Phi_{j}(\xi)(z-\xi)+(z-\xi)^{\perp} B_{j}(\xi)(z-\xi) e_{n}
$$

where matrices $\Phi_{j}(\xi), B_{j}(\xi)$ are $C^{1}$-smooth on $\Gamma_{j}$, and $e_{n}=(0, \ldots, 0,1)$.

2. Let $\psi_{j}(\xi, \cdot)$ be an inverse map of $\varphi_{j}(\xi, \cdot)$, and let $J_{j}(\xi, \cdot)$ be a complex Jacobian of $\psi_{j}$. Then

$$
\begin{gathered}
\sup _{\tau \in \Omega_{\varepsilon} \backslash \bar{\Omega}_{\varepsilon}}\left|J_{j}(\xi, \cdot)-J_{j}\left(\xi^{\prime}, \cdot\right)\right| \lesssim\left|\xi-\xi^{\prime}\right|, \\
\sup _{\tau \in \Omega_{\varepsilon} \backslash \bar{\Omega}_{\varepsilon}}\left|\psi_{j}(\xi, \cdot)-\psi_{j}\left(\xi^{\prime}, \cdot\right)\right| \lesssim\left|\xi-\xi^{\prime}\right| .
\end{gathered}
$$

Note that real Jacobian is then equal to $\left|J_{j}(\xi, \cdot)\right|^{2}=J_{j}(\xi, \cdot) \overline{J_{j}(\xi, \cdot)}$.

3. There exist constants $c, \varepsilon_{0}>0$ such that for $0<\eta, \varepsilon<\varepsilon_{0}$

$$
\varphi_{j}\left(\xi, D^{e}(\xi, \eta, \varepsilon)\right) \subseteq D_{0}(c \eta, c \varepsilon), \quad \psi_{j}\left(\xi, D_{0}(\eta, \varepsilon) \subseteq D^{e}(\xi, c \eta, c \varepsilon) .\right.
$$


Proof. Let $\xi \in \partial \Omega$, by linear change of coordinates $z^{\prime}=(z-\xi) \Phi(\xi)$ we could obtain the following form for function $\rho$

$$
\begin{aligned}
\rho(z)=\rho(\xi+ & \left.\Phi^{-1}(\xi) z^{\prime}\right) \\
& =2 \operatorname{Re}\left(z_{n}^{\prime}\right)+\sum_{j, k=1}^{n} A_{j k}^{1}(\xi) z_{j}^{\prime} \bar{z}_{k}^{\prime}+\operatorname{Re} \sum_{j, k=1}^{n} A_{j k}^{2}(\xi) z_{j}^{\prime} z_{k}^{\prime}+O\left(\left|z^{\prime}\right|^{3}\right) .
\end{aligned}
$$

Setting $z_{n}^{\prime \prime}=z_{n}^{\prime}+A_{j k}^{2} z_{j}^{\prime} z_{k}^{\prime}$ and $z_{j}^{\prime \prime}=z_{j}^{\prime}, 1 \leq j \leq n-1$, we have (see [12])

$$
\rho\left(z^{\prime \prime}\right)=2 \operatorname{Re}\left(z_{n}^{\prime}\right)+\sum_{j, k=1}^{n} A_{j k}^{1}(\xi) z_{j}^{\prime \prime} \bar{z}_{k}^{\prime \prime}+O\left(\left|z^{\prime \prime}\right|^{3}\right) .
$$

Denote $B(\xi)=\Phi(\xi)^{\perp} A^{2}(\xi) \Phi(\xi)$, then

$$
\varphi(\xi, z)=\Phi(\xi)(z-\xi)+(z-\xi)^{\perp} B(\xi)(z-\xi) e_{n} .
$$

We choose $\Gamma_{j}$ such that the matrix $\Phi(\xi)$ could be defined on $\Gamma_{j}$ smoothly, this choice we denote as $\Phi_{j}$, and the change corresponding to this matrix as $\varphi_{j}$

$$
\varphi_{j}(\xi, z)=\Phi_{j}(\xi)(z-\xi)+(z-\xi)^{\perp} B_{j}(\xi)(z-\xi) e_{n}
$$

Thus mappings $\varphi_{j}$ satisfy the first condition. Easily, the second condition also holds.

The last condition (14) follows immediately from lemma 4.3. This ends the proof of the theorem.

Further we will assume, that the covering $\bar{\Omega}_{\varepsilon} \backslash \Omega_{-\varepsilon} \subset \bigcup_{j=1}^{N} \Gamma_{j}$ and maps $\varphi_{j}, \psi_{j}$ are chosen by the theorem 4.4. For covering $\left\{\Gamma_{j}\right\}$ we consider a smooth decomposition of identity on $\partial \Omega$ :

$$
\chi_{j} \in C^{\infty}\left(\Gamma_{j}\right), 0 \leq \chi_{j} \leq 1, \operatorname{supp} \chi_{j} \subset \Gamma_{j}, \sum_{j=1}^{N} \chi_{j}(z)=1, z \in \partial \Omega .
$$

Fix parameters $0<\varepsilon, \eta<\varepsilon_{0}$, denote $D_{0}=D_{0}(\eta, \varepsilon)$. Then by (14)

$$
D^{e}(z)=\varphi_{j}\left(z, D^{e}(z, \eta / c, \varepsilon / c)\right) \subset D_{0}
$$


and

$$
\begin{aligned}
& I_{l}(g, z)^{2} \\
& \quad=\sum_{j=1}^{N} \chi_{j}(z) \int_{D^{e}(z)}\left|\int_{\partial \Omega} \frac{g(w) J_{j}(z, \tau) d S(w)}{\left\langle\partial \rho\left(\psi_{j}(z, \tau)\right), \psi_{j}(z, \tau)-w\right\rangle^{n+l}}\right|^{2} \frac{d \mu(\tau)}{\operatorname{Re}\left(\tau_{n}\right)^{n-2 l+1}} \\
& \quad \lesssim \sum_{j=1}^{N} \int_{D_{0}}\left|\int_{\partial \Omega} \frac{g(w) \chi_{j}^{1 / 2}(z) J_{j}(z, \tau) d S(w)}{\left\langle\partial \rho\left(\psi_{j}(z, \tau)\right), \psi_{j}(z, \tau)-w\right\rangle^{n+l}}\right|^{2} \frac{d \mu(\tau)}{\operatorname{Re}\left(\tau_{n}\right)^{n-2 l+1}} .
\end{aligned}
$$

We will consider the function

$$
K_{j}(z, w)(\tau)=\frac{\chi_{j}^{1 / 2}(z) J_{j}(z, \tau)}{\left\langle\partial \rho\left(\psi_{j}(z, \tau)\right), \psi_{j}(z, \tau)-w\right\rangle^{n+1}}
$$

as a map $\partial \Omega \times \partial \Omega \rightarrow \mathscr{L}\left(\mathbb{C}, L^{2}\left(D_{0}, d \nu_{l}\right)\right)$, such that its values are operator of multiplication from $\mathbb{C}$ to $L^{2}\left(D_{0}, d \nu_{l}\right)$, where $d \nu_{l}(\tau)=\frac{d \mu(\tau)}{\operatorname{Im}\left(\tau_{n}\right)^{n-2 l+1}}$ is a measure on the region $D_{0}$. Throughout the proof of the theorem $4.1 j, l$ will be fixed integers and the norm of function $F$ in the space $L^{2}\left(D_{0}, d \nu_{l}\right)$ will be denoted as $\|F\|$.

We will show that integral operator $T_{j}$ defined by kernel $K_{j}$ is bounded on $L^{p}$. To prove this we apply $T 1$-theorem for transformations with operator-valued kernels formulated by Hytönen and Weis in [7], taking in account that in our case concerned spaces are Hilbert. Some details of the proof are similar to the proof of the boundedness of operator Cauchy-Leray-Fantappiè $K_{\Omega}$ for lineally convex domains introduced in [13]. Below we formulate the T1-theorem, adapted to our context.

Definition 4.2. We say that the function $f \in C_{0}^{\infty}(\partial \Omega)$ is a normalized bumpfunction, associated with the quasiball $B\left(w_{0}, r\right)$ if supp $f \subset B\left(w_{0}, r\right),|f| \leq 1$, and

$$
|f(\xi)-f(z)| \leq \frac{d(\xi, z)^{\gamma}}{r^{\gamma}}, \xi, z \in \partial \Omega .
$$

The set of bump-functions associated with $B\left(w_{0}, r\right)$ is denoted as $A\left(\gamma, w_{0}, r\right)$. 
Theorem 4.5. Let $K: \partial \Omega \times \partial \Omega \rightarrow \mathscr{L}\left(\mathbb{C}, L^{2}\left(D_{0}, d \nu_{l}\right)\right)$ verify the estimates

$$
\begin{aligned}
& \|K(z, w)\| \lesssim \frac{1}{d(z, w)^{n}} \\
& \|K(z, w)-K(\xi, w)\| \lesssim \frac{d(z, \xi)^{\gamma}}{d(z, w)^{n+\gamma}}, \quad d(z, w)>C d(z, \xi) \\
& \left\|K(z, w)-K\left(z, w^{\prime}\right)\right\| \lesssim \frac{d\left(w, w^{\prime}\right)^{\gamma}}{d(z, w)^{n+\gamma}}, \quad d(z, w)>C d\left(w, w^{\prime}\right)
\end{aligned}
$$

for $\xi, z, w \in \partial \Omega$ and some constant $C>0$.

Assume that operator $T: \mathscr{S}(\partial \Omega) \rightarrow \mathscr{S}^{\prime}\left(\partial \Omega, \mathscr{L}\left(\mathbb{C}, L^{2}\left(D_{0}, d \nu_{l}\right)\right)\right)$ with kernel $K$ verify the following conditions.

- $T 1, T^{\prime} 1 \in \operatorname{BMO}\left(\partial \Omega, L^{2}\left(D_{0}, d \nu_{l}\right)\right)$, where $T^{\prime}$ is formally adjoint operator.

- Operator T satisfies the weak boundedness property, that is for every pair of normalized bump-functions $f, g \in A\left(\gamma, w_{0}, r\right)$ we have

$$
\|\langle g, T f\rangle\| \leq C r^{-n} .
$$

Then $T \in \mathscr{L}\left(L^{p}(\partial \Omega), L^{p}\left(\partial \Omega, L^{2}\left(D_{0}, d \nu_{l}\right)\right)\right.$ for every $p \in(1, \infty)$.

In the following four lemmas we will prove that kernels $K_{j}$ and corresponding operators $T_{j}$ satisfy the conditions of the $T 1$-theorem. In particular, in lemmas 4.7,4.9 we prove that $T 1, T^{\prime} 1 \in L^{\infty}\left(\partial \Omega, L^{2}\left(D_{0}, d \nu_{l}\right)\right) \subset \operatorname{BMO}\left(\partial \Omega, L^{2}\left(D_{0}, d \nu_{l}\right)\right)$.

Lemma 4.6. The kernel $K_{j}$ verify estimates (17-19).

Proof. By lemma 3.2 we have $|\langle\partial \rho(\tau), \tau-w\rangle| \asymp \rho(\tau)+|\langle\partial \rho(z), z-w\rangle|$, $z, w \in \partial \Omega, \tau \in D^{e}(z, c \eta, c \varepsilon)$. Thus

$$
\begin{aligned}
\left\|K_{j}(z, w)\right\|^{2} & =\int_{D_{0}}\left|K_{j}(z, w)(\tau)\right|^{2} d \nu_{l}(\tau) \lesssim \int_{D^{e}(z, c \eta, c \varepsilon)} \frac{d \nu_{l}(\tau)}{|\langle\partial \rho(\tau), \tau-w\rangle|^{2 n+2 l}} \\
& \lesssim \int_{D^{e}(z, c \eta, c \varepsilon)} \frac{1}{(\rho(\tau)+|\langle\partial \rho(z), z-w\rangle|)^{2 n+2 l}} \frac{d \mu(\tau)}{\rho(\tau)^{n-2 l+1}} \\
\lesssim & \int_{0}^{\infty} \frac{t^{2 l-1} d t}{(t+|\langle\partial \rho(z), z-w\rangle|)^{2 n+2 l}} \lesssim \frac{1}{|\langle\partial \rho(z), z-w\rangle|^{2 n}} \lesssim \frac{1}{d(z, w)^{2 n}} .
\end{aligned}
$$


Similarly,

$$
\begin{aligned}
\| K_{j}(z, w)- & K_{j}\left(z, w^{\prime}\right) \|^{2} \\
& \lesssim \int_{D^{e}(z, c \eta, c \varepsilon)}\left|\frac{1}{\langle\partial \rho(\tau), \tau-w\rangle^{n+l}}-\frac{1}{\left\langle\partial \rho(\tau), \tau-w^{\prime}\right\rangle^{n+l}}\right|^{2} d \nu_{l}(\tau) .
\end{aligned}
$$

Denote $\hat{\tau}=\operatorname{pr}_{\partial \Omega}(\tau)$, then

$$
\begin{aligned}
& |\langle\partial \rho(\tau), \tau-w\rangle| \lesssim \rho(\tau)+|\langle\partial \rho(\hat{\tau}), \hat{\tau}-w\rangle| \\
& \quad \lesssim \rho(\tau)+|\langle\partial \rho(z), z-w\rangle|+|\langle\partial \rho(\hat{\tau}), \hat{\tau}-z\rangle| \lesssim \rho(\tau)+|\langle\partial \rho(z), z-w\rangle|,
\end{aligned}
$$

which combined with lemma 3.2 and estimate

$$
d\left(w, w^{\prime}\right)=\left|\left\langle\partial \rho(w), w-w^{\prime}\right\rangle\right|<C|\langle\partial \rho(z), z-w\rangle|=C d(z, w)
$$

implies

$$
\begin{aligned}
|\langle\partial \rho(\tau), \tau-w\rangle| \asymp \rho(\tau)+|\langle\partial \rho(z), z-w\rangle| \asymp \rho(\tau)+ & \left|\left\langle\partial \rho(z), z-w^{\prime}\right\rangle\right| \\
& \asymp\left|\left\langle\partial \rho(\tau), \tau-w^{\prime}\right\rangle\right| .
\end{aligned}
$$

Next, we have

$$
\begin{gathered}
\left|\left\langle\partial \rho(\tau), \tau-w^{\prime}\right\rangle-\langle\partial \rho(\tau), \tau-w\rangle\right|=\left|\langle\partial \rho(\tau), \hat{\tau}-w\rangle-\left\langle\partial \rho(\tau), \hat{\tau}-w^{\prime}\right\rangle\right| \\
\leq\left|\left\langle\partial \rho(\tau)-\partial \rho(\hat{\tau}), w-w^{\prime}\right\rangle\right|+\left|\langle\partial \rho(\hat{\tau}), \hat{\tau}-w\rangle-\left\langle\partial \rho(\hat{\tau}), \hat{\tau}-w^{\prime}\right\rangle\right| \\
\lesssim \rho(\tau)\left|\left\langle\partial \rho(w), w-w^{\prime}\right\rangle\right|^{1 / 2}+|\langle\partial \rho(\hat{\tau}), \hat{\tau}-w\rangle|^{1 / 2}\left|\left\langle\partial \rho(w), w-w^{\prime}\right\rangle\right|^{1 / 2} \\
\lesssim|\langle\partial \rho(\tau), \tau-w\rangle|^{1 / 2}\left|\left\langle\partial \rho(w), w-w^{\prime}\right\rangle\right|^{1 / 2}
\end{gathered}
$$

Hence,

$$
\begin{aligned}
& \left\|K_{j}(z, w)-K_{j}\left(z, w^{\prime}\right)\right\|^{2} \lesssim \int_{D^{e}(z, c \eta, c \varepsilon)} \frac{\left|\left\langle\partial \rho(w), w-w^{\prime}\right\rangle\right|}{|\langle\partial \rho(\tau), \tau-w\rangle|^{2 n+2 l+1}} d \nu_{l}(\tau) \\
& \lesssim \int_{0}^{\infty} \frac{\left|\left\langle\partial \rho(w), w-w^{\prime}\right\rangle\right| t^{2 l-1} d t}{(t+|\langle\partial \rho(z), z-w\rangle|)^{2 n+2 l+1}} \lesssim \frac{\left|\left\langle\partial \rho(w), w-w^{\prime}\right\rangle\right|}{|\langle\partial \rho(z), z-w\rangle|^{2 n+1}}=\frac{d\left(w, w^{\prime}\right)}{d(z, w)^{2 n+1}}
\end{aligned}
$$

The last inequality (19) is a bit harder to prove. 
Let $z, \xi, w \in \partial \Omega, C d(z, \xi)<d(z, w)$, and estimate the value

$$
A=\left|\left\langle\partial \rho\left(\psi_{j}(z, \tau)\right), \psi_{j}(z, \tau)-w\right\rangle-\left\langle\partial \rho\left(\psi_{j}(\xi, \tau)\right), \psi_{j}(\xi, \tau)-w\right\rangle\right| .
$$

Denote $\tau_{z}=\psi_{j}(z, \tau), \tau_{\xi}=\psi_{j}(\xi, \tau)$, then by (11)

$$
\begin{aligned}
\tau=\Phi(z)\left(\tau_{z}-z\right)+i\left(\tau_{z}-z\right)^{T} B(z) & \left(\tau_{z}-z\right) e_{n} \\
& =\Phi(\xi)\left(\tau_{\xi}-\xi\right)+i\left(\tau_{\xi}-\xi\right)^{T} B(\xi)\left(\tau_{\xi}-\xi\right) e_{n},
\end{aligned}
$$

whence denoting $\Psi(z)=\Phi(z)^{-1}$ and introducing $L(z, \xi, \tau)$ we obtain

$$
\begin{aligned}
\tau_{z} & =z+\Psi(z) \tau-\left(\tau_{z}-z\right)^{T} B(z)\left(\tau_{z}-z\right) \Psi(z) e_{n}, \\
\tau_{\xi} & =\xi+\Psi(\xi) \tau-\left(\tau_{\xi}-\xi\right)^{T} B(\xi)\left(\tau_{\xi}-\xi\right) \Psi(\xi) e_{n}, \\
\tau_{z}-\tau_{\xi} & =z-\xi+(\Psi(z)-\Psi(\xi)) \tau+L(z, \xi, \tau) e_{n} .
\end{aligned}
$$

Note, that norms of matrices $\|\Psi(\xi)\|$ are bounded, thus

$$
\begin{gathered}
|L(z, \xi, \tau)| \leq\left|\left(\tau_{z}-z\right)^{T} B(z)\left(\tau_{z}-z\right)(\Psi(z)-\Psi(\xi))\right| \\
+\left|\left(\tau_{z}-z\right)^{T} B(z)\left(\tau_{z}-z\right)-\left(\tau_{\xi}-\xi\right)^{T} B(\xi)\left(\tau_{\xi}-\xi\right)\right|\|\Psi(\xi)\| \\
\quad \lesssim|z-\xi|\left|\tau_{z}-z\right|^{2}+\left|\left(\tau_{z}-z-\tau_{\xi}+\xi\right)^{T} B(z)\left(\tau_{z}-z\right)\right| \\
\quad+\left|\left(\tau_{\xi}-\xi\right)^{T} B(z)\left(\tau_{z}-z\right)-\left(\tau_{\xi}-\xi\right)^{T} B(\xi)\left(\tau_{\xi}-\xi\right)\right| \\
\lesssim|z-\xi|\left|\tau_{z}-z\right|^{2}+|z-\xi||\tau|+\left|\left((\Psi(z)-\Psi(\xi)) \tau+L(z, \xi, \tau) e_{n}\right)^{T} B(z)\left(\tau_{z}-z\right)\right| \\
+\left|\left(\tau_{\xi}-\xi\right)^{T}(B(z)-B(\xi))\left(\tau_{z}-z\right)\right|+\left|\left(\tau_{\xi}-\xi\right)^{T} B(\xi)\left(\tau_{z}-z-\tau_{\xi}-\xi\right)\right| \\
\lesssim|z-\xi|\left|\tau_{z}-z\right|^{2}+|z-\xi||\tau|+|\tau||L(z, \xi, \tau)|+|z-\xi||\tau|^{2}+|\tau| L(z, \xi, \tau) .
\end{gathered}
$$

Choosing $\varepsilon>0$ small enough we get $|\tau| \leq \eta\left|\operatorname{Im}\left(\tau_{n}\right)\right|+(1+\eta)\left|\operatorname{Im}\left(\tau_{n}\right)\right| \leq 3 \varepsilon$ and $|L(z, \xi, \tau)| \lesssim d(z, \xi)^{1 / 2}|\tau|$, for $\tau \in D_{0}=D_{0}(\eta, \varepsilon)$. Hence,

$$
\begin{gathered}
A \leq\left|\left\langle\partial \rho\left(\tau_{z}\right)-\partial \rho\left(\tau_{\xi}\right), \tau_{z}-w\right\rangle\right|+\left|\left\langle\partial \rho\left(\tau_{\xi}\right), \tau_{z}-w\right\rangle\right| \\
\lesssim\left|\tau_{z}-\tau_{\xi}\right|\left(\rho\left(\tau_{z}\right)+d(z, w)^{1 / 2}\right)+\left|\left\langle\partial \rho\left(\tau_{z}\right)-\partial \rho\left(\tau_{\xi}\right), z-\xi\right\rangle\right|+|\langle\partial \rho(z), z-\xi\rangle| \\
+\left|\left\langle\partial \rho\left(\tau_{\xi}\right),(\Psi(z)-\Psi(\xi)) \tau\right\rangle\right|+\left|\left\langle\partial \rho\left(\tau_{\xi}\right), L(z, \xi, \tau)\right\rangle\right| \lesssim d(z, \xi)^{1 / 2} d\left(\tau_{z}, w\right)+ \\
\left|\tau_{z}-\xi\right||z-\xi|+d(z, \xi)+|z-\xi||\tau|+|L(z, \xi, \tau)| \lesssim d(z, \xi)+d(z, \xi)^{1 / 2} d(z, w)^{1 / 2} \\
\lesssim d(z, \xi)^{1 / 2} d(z, w)^{1 / 2}
\end{gathered}
$$


Combining this estimate with inequality $\left|\left\langle\partial \rho\left(\tau_{z}\right), \tau_{z}-w\right\rangle\right| \asymp\left|\left\langle\partial \rho\left(\tau_{\xi}\right), \tau_{\xi}-w\right\rangle\right|$ we obtain

$$
\begin{gathered}
\left\|K_{j}(z, w)-K_{j}(\xi, w)\right\|^{2} \lesssim \int_{D^{e}(z, c \eta, c \varepsilon)} \frac{\left|\chi_{j}(z)^{1 / 2}-\chi_{j}(\xi)^{1 / 2}\right|^{2}}{|\langle\partial \rho(\tau), \tau-w\rangle|^{2 n+2 l}} \frac{d \mu(\tau)}{\rho(\tau)^{n-2 l+1}} \\
+\chi_{j}(\xi) \int_{D_{0}} \frac{|\langle\partial \rho(z), z-\xi\rangle||\langle\partial \rho(z), z-w\rangle|}{\left|\left\langle\partial \rho\left(\tau_{z}\right), \tau_{z}-w\right\rangle\right|^{2 n+4}} \frac{d \mu(\tau)}{\operatorname{Re}\left(\tau_{n}\right)^{n-2 l+1}} \\
\lesssim \frac{|\langle\partial \rho(z), z-\xi\rangle|}{|\langle\partial \rho(z), z-w\rangle|^{2 n}}+\frac{|\langle\partial \rho(z), z-\xi\rangle|}{|\langle\partial \rho(z), z-w\rangle|^{2 n+1}} \lesssim \frac{|\langle\partial \rho(z), z-\xi\rangle|}{|\langle\partial \rho(z), z-w\rangle|^{2 n+1}} \\
\lesssim \frac{d(z, \xi)}{d(z, w)^{2 n+1}} .
\end{gathered}
$$

Lemma 4.7. Let $\tau_{z}=\psi_{j}(z, \tau)$, then

$$
\left\|\int_{\partial \Omega} \frac{d S(w)}{\left\langle\partial \rho\left(\tau_{z}\right), \tau_{z}-w\right\rangle^{n+l}}\right\| \lesssim 1
$$

and, consequently, $\left\|T_{j}(1)\right\| \lesssim 1$.

Proof. The function $\left\langle\partial \rho\left(\tau_{z}\right), \tau_{z}-w\right\rangle$ is holomorphic in $\Omega$ with respect to $w$, then

$$
T_{j}(1)(\tau)=\int_{\partial \Omega} \frac{\chi_{j}(z)^{1 / 2} J_{j}(z, \tau) d S(w)}{\left\langle\partial \rho\left(\tau_{z}\right), \tau_{z}-w\right\rangle^{n+l}}=\int_{\Omega} \frac{\chi_{j}(z)^{1 / 2} J_{j}(z, \tau) d V(w)}{\left\langle\partial \rho\left(\tau_{z}\right), \tau_{z}-w\right\rangle^{n+l}} .
$$

Analogously to lemma 3.2 we have $\left|\left\langle\partial \rho\left(\tau_{z}\right), \tau_{z}-w\right\rangle\right| \asymp \operatorname{Im}\left(\tau_{n}\right)+|\rho(w)|+$ $|\langle\partial \rho(z), z-\hat{w}\rangle|$, where $\hat{w}=\operatorname{pr}_{\partial \Omega}(w)$.

Hence,

$$
\begin{aligned}
&\left|T_{j}(1)(\tau)\right| \lesssim \int_{\Omega} \frac{d \mu(z)}{\left|\left\langle\partial \rho\left(\tau_{z}\right), \tau_{z}-w\right\rangle\right|^{n+l}} \\
& \lesssim \int_{0}^{T} d t \int_{\partial \Omega_{t}} \frac{d \sigma_{t}}{\left(t+\operatorname{Im}\left(\tau_{n}\right)+|\langle\partial \rho(z), z-\hat{w}\rangle|\right)^{n+l}} \\
& \lesssim \int_{0}^{T} d t \int_{0}^{\infty} \frac{v^{n-1} d v}{\left(t+\operatorname{Re}\left(\tau_{n}\right)+v\right)^{n+l}} \lesssim \int_{0}^{T} \frac{d t}{\left(t+\operatorname{Re}\left(\tau_{n}\right)\right)^{l}} \\
& \quad \lesssim\left(\operatorname{Re}\left(\tau_{n}\right)\right)^{1-l} \ln \left(1+\frac{1}{\operatorname{Re}\left(\tau_{n}\right)}\right),
\end{aligned}
$$


and

$$
\begin{aligned}
\int_{D_{0}}\left|T_{j}(1)(\tau)\right|^{2} d \nu_{l}(\tau) \lesssim \int_{D_{0}}\left(\operatorname{Re}\left(\tau_{n}\right)\right)^{2-2 l} \ln ^{2}(1 & \left.+\frac{1}{\operatorname{Re}\left(\tau_{n}\right)}\right) d \nu_{l}(\tau) \\
& \lesssim \int_{0}^{\varepsilon} \ln ^{2}\left(1+\frac{1}{s}\right) s d s \lesssim 1 .
\end{aligned}
$$

This finishes the proof of the lemma.

Lemma 4.8. $\left\|T_{j}^{\prime}(1)\right\| \lesssim 1$.

Proof. Consider

$$
\begin{aligned}
& \overline{T_{j}^{\prime}(1)}(w)(\tau)=\int_{\partial \Omega} \frac{\chi_{j}(z)^{1 / 2} J_{j}(z, \tau) d S(z)}{\left\langle\partial \rho\left(\tau_{z}\right), \tau_{z}-w\right\rangle^{n+l}} \\
= & \int_{\partial \Omega} \frac{\chi_{j}(z)^{1 / 2} J_{j}(z, \tau)\left(d S(z)-d S\left(\tau_{z}\right)\right)}{\left\langle\partial \rho\left(\tau_{z}\right), \tau_{z}-w\right\rangle^{n+l}}+\int_{\partial \Omega} \frac{\chi_{j}(z)^{1 / 2} J_{j}(z, \tau) d S\left(\tau_{z}\right)}{\left\langle\partial \rho\left(\tau_{z}\right), \tau_{z}-w\right\rangle^{n+l}}=L_{1}+L_{2} .
\end{aligned}
$$

Note that $\left|z-\tau_{z}\right| \lesssim \operatorname{Re}\left(\tau_{n}\right)$, therefore $|d S(z)-d S(\psi(z, \tau))| \lesssim \operatorname{Re}\left(\tau_{n}\right) d \sigma(z)$ and

$$
\begin{aligned}
\left|L_{1}\right| \lesssim \int_{\partial \Omega} \frac{\operatorname{Re}\left(\tau_{n}\right) d \sigma(z)}{\left|\left\langle\partial \rho\left(\tau_{z}\right), \tau_{z}-w\right\rangle\right|^{n+l}} \lesssim \frac{\operatorname{Re}\left(\tau_{n}\right) d \sigma(z)}{\left(\operatorname{Re}\left(\tau_{n}\right)+|\langle\partial \rho(z), z-w\rangle|\right)^{n+l}} \\
\quad \lesssim \int_{0}^{\infty} \frac{\operatorname{Re}\left(\tau_{n}\right) v^{n-1} d v}{\left(\operatorname{Re}\left(\tau_{n}\right)+v\right)^{n+l}} \lesssim \frac{1}{\operatorname{Re}\left(\tau_{n}\right)^{l-1}} .
\end{aligned}
$$

Thus we get

$$
\int_{D_{0}}\left|L_{1}\right|^{2} d \nu_{l}(\tau) \lesssim \int_{D_{0}} \frac{1}{\operatorname{Re}\left(\tau_{n}\right)^{2 l-2}} \frac{d \mu(\tau)}{\operatorname{Re}\left(\tau_{n}\right)^{n-2 l+1}} \lesssim \int_{0}^{\varepsilon} \frac{t^{n} d t}{t^{n-1}} \lesssim 1
$$

To estimate $L_{2}$ we recall that $d_{\xi} \frac{d S(\xi)}{\langle\partial \rho(\xi), \xi-z\rangle^{n}}=0, z \in \partial \Omega, \xi \in \mathbb{C}^{n} \backslash \Omega$, and consequently

$$
\begin{aligned}
& d_{\xi} \frac{d S(\xi)}{\langle\partial \rho(\xi), \xi-z\rangle^{n+l}}=\frac{(\bar{\partial} \partial \rho(\xi))^{n}}{\langle\partial \rho(\xi), \xi-z\rangle^{n+l}} \\
& \quad-(n+l) \frac{\left(\bar{\partial}_{\xi}(\langle\partial \rho(\xi), \xi-z\rangle) \wedge \bar{\partial} \partial \rho(\xi)\right)^{n-1}}{\langle\partial \rho(\xi), \xi-z\rangle^{n+l}}=-\frac{l}{n} \frac{d V(\xi)}{\langle\partial \rho(\xi), \xi-z\rangle^{n+l}} .
\end{aligned}
$$


By Stokes' theorem we obtain

$$
\begin{aligned}
L_{2} & =\int_{\partial \Omega} \frac{\chi_{j}(z)^{1 / 2} J_{j}(z, \tau) d S\left(\tau_{z}\right)}{\left\langle\partial \rho\left(\tau_{z}\right), \tau_{z}-w\right\rangle^{n+l}} \\
& =\int_{\Omega_{\varepsilon_{1}} \backslash \Omega} \frac{\bar{\partial}_{z}\left(\chi_{j}(z)^{1 / 2} J_{j}(z, \tau)\right) \wedge d S\left(\tau_{z}\right)}{\left\langle\partial \rho\left(\tau_{z}\right), \tau_{z}-w\right\rangle^{n+l}}-\frac{l}{n} \int_{\Omega_{\varepsilon_{1} \backslash \Omega}} \frac{\chi_{j}(z)^{1 / 2} J_{j}(z, \tau) d V\left(\tau_{z}\right)}{\left\langle\partial \rho\left(\tau_{z}\right), \tau_{z}-z\right\rangle^{n+l}}
\end{aligned}
$$

Again similarly to lemma 3.2 we have $\left|\left\langle\partial \rho\left(\tau_{z}\right), \tau_{z}-w\right\rangle\right| \asymp \operatorname{Im}\left(\tau_{n}\right)+\rho(z)+$ $|\langle\partial \rho(\hat{z}), \hat{z}-w\rangle|$, where $\hat{z}=\operatorname{pr}_{\partial \Omega}(z)$, and the estimate $\left\|L_{2}\right\| \lesssim 1$ is proven analogously to lemma 4.7. Combining this with the estimate (23) we get $\left\|T_{j}(1)\right\| \lesssim 1$.

Lemma 4.9. Operator $T_{j}$ is weakly bounded.

Proof. Let $f, g \in A\left(\frac{1}{2}, w_{0}, r\right)$, denote again $\tau_{z}=\psi_{j}(z, \tau)$, then

$$
\left\|\left\langle g, T_{j} f\right\rangle\right\|^{2} \lesssim \int_{D_{0}} d \nu_{l}(\tau)\left(\int_{B\left(w_{0}, r\right)}|g(z)| d S(z)\left|\int_{B\left(w_{0}, r\right)} \frac{f(w) d S(w)}{\left\langle\partial \rho\left(\tau_{z}\right), \tau_{z}-w\right\rangle^{n+l}}\right|\right)^{2} .
$$

Denote $t:=\inf _{w \in \partial \Omega}\left|\left\langle\partial \rho\left(\tau_{z}\right), \tau_{z}-w\right\rangle\right|$ and introduce the set

$$
W(z, \tau, r):=\left\{w \in \partial \Omega:\left|\left\langle\partial \rho\left(\tau_{z}\right), \tau_{z}-w\right\rangle\right|<t+r\right\} .
$$

Note that supp $f \subset B\left(w_{0}, r\right) \subset W(z, \tau, c r) \subset B\left(z, c^{2} r\right)$ for some $c>0$, therefore,

$$
\begin{aligned}
& \left|\int_{B\left(w_{0}, r\right)} \frac{f(w) d S(w)}{\left\langle\partial \rho\left(\tau_{z}\right), \tau_{z}-w\right\rangle^{n+l}}\right| \\
& =\left|\int_{W(z, \tau, c r)} \frac{f(w) d S(w)}{\left\langle\partial \rho\left(\tau_{z}\right), \tau_{z}-w\right\rangle^{n+l}}\right| \lesssim \int_{W(z, \tau, c r)} \frac{|f(z)-f(w)| d S(w)}{\mid\left\langle\partial \rho\left(\tau_{z}\right), \tau_{z}-\left.w\right|^{n+l}\right.} \\
& +|f(z)|\left(\left|\int_{\partial \Omega \backslash W(z, \tau, c r)} \frac{d S(w)}{\left\langle\partial \rho\left(\tau_{z}\right), \tau_{z}-w\right\rangle^{n+l}}\right|+\left|\int_{\partial \Omega} \frac{d S(w)}{\left\langle\partial \rho\left(\tau_{z}\right), \tau_{z}-w\right\rangle^{n+l}}\right|\right) \\
& =L_{1}(z, \tau)+|f(z)|\left(L_{2}(z, \tau)+L_{3}(z, \tau)\right) .
\end{aligned}
$$


It follows from the estimate $|f(z)-f(w)| \leq \sqrt{v(w, z) / r}$ that

$$
\begin{gathered}
L_{1}(z, \tau) \lesssim \frac{1}{\sqrt{r}} \int_{B\left(z, c^{2} r\right)} \frac{v(w, z)^{1 / 2}}{\left(\operatorname{Re}\left(\tau_{n}\right)+v(w, z)\right)^{n+l}} \lesssim \frac{1}{\sqrt{r}} \int_{0}^{c^{2} r} \frac{t^{n-1 / 2} d t}{\left(\operatorname{Re}\left(\tau_{n}\right)+t\right)^{n+l}} \\
\lesssim \frac{1}{\sqrt{r}} \int_{0}^{c^{2} r} \frac{d t}{\left(\operatorname{Re}\left(\tau_{n}\right)+t\right)^{l+1 / 2}} \lesssim \frac{1}{\sqrt{r}}\left(\frac{1}{\operatorname{Re}\left(\tau_{n}\right)^{l-1 / 2}}-\frac{1}{\left(\operatorname{Re}\left(\tau_{n}\right)+r\right)^{l-1 / 2}}\right) \\
=\frac{1}{\sqrt{r}} \frac{\left(\operatorname{Re}\left(\tau_{n}\right)+r\right)^{l-1 / 2}-r^{l-1 / 2}}{\operatorname{Re}\left(\tau_{n}\right)^{l-1 / 2}\left(\operatorname{Re}\left(\tau_{n}\right)+r\right)^{l-1 / 2}} \lesssim \frac{1}{\sqrt{r}} \frac{\left(\operatorname{Re}\left(\tau_{n}\right)+r\right)^{2 l-1}-r^{2 l-1}}{\operatorname{Im}\left(\tau_{n}\right)^{l-1 / 2}\left(\operatorname{Re}\left(\tau_{n}\right)+r\right)^{2 l-1}} \\
\lesssim \frac{1}{\sqrt{r}} \frac{r \operatorname{Re}\left(\tau_{n}\right)^{2 l-2}+r^{2 l-1}}{\operatorname{Re}\left(\tau_{n}\right)^{l-1 / 2}\left(\operatorname{Re}\left(\tau_{n}\right)+r\right)^{2 l-1}} .
\end{gathered}
$$

Estimating the $L^{2}\left(D_{0}, d \nu_{l}\right)$-norm of the function $L_{1}(z, \tau)$, we obtain

$$
\begin{aligned}
& \int_{D_{0}(\tau)} L_{1}(z, \tau)^{2} d \nu_{l}(\tau) \\
& \lesssim \int_{D_{0}(\tau)}\left(\frac{r \operatorname{Re}\left(\tau_{n}\right)^{2 l-3}}{\left(\operatorname{Re}\left(\tau_{n}\right)+r\right)^{4 l-2}}+\frac{r^{4 l-3}}{\operatorname{Re}\left(\tau_{n}\right)^{2 l-1}\left(\operatorname{Re}\left(\tau_{n}\right)+r\right)^{4 l-2}}\right) \frac{d \mu(\tau)}{\operatorname{Re}\left(\tau_{n}\right)^{n-2 l+1}} \\
& \lesssim r \int_{0}^{\infty} \frac{s^{4 l-4}}{(s+r)^{4 l-2}} d s+r^{4 l-3} \int_{0}^{\infty} \frac{d s}{(s+r)^{4 l-2}} \lesssim 1
\end{aligned}
$$

To estimate the second summand $L_{2}$ we apply the Stokes theorem to the domain

$$
W_{0}=\left\{w \in \Omega:\left|\left\langle\partial \rho\left(\tau_{z}\right), \tau_{z}-w\right\rangle\right|>t+c r\right\}
$$

and to the form $\frac{d S(w)}{\left\langle\partial \rho\left(\tau_{z}\right), \tau_{z}-w\right\rangle^{n+l}}$

$$
\begin{gathered}
\int_{\partial \Omega \backslash W(z, \tau, c r)} \frac{d S(w)}{\left\langle\partial \rho\left(\tau_{z}\right), \tau_{z}-w\right\rangle^{n+l}}=\int_{W_{0}} \frac{d V(w)}{\left\langle\partial \rho\left(\tau_{z}\right), \tau_{z}-w\right\rangle^{n+l}} \\
-\int_{\substack{w \in \Omega \\
\left|v\left(\tau_{z}, w\right)\right|=t+c r}} \frac{d S(w)}{\left\langle\partial \rho\left(\tau_{z}\right), \tau_{z}-w\right\rangle^{n+l}} \\
=L_{4}-\frac{1}{(t+c r)^{2 n+2 l}} \int_{\substack{w \in \Omega \\
\left|v\left(\tau_{z}, w\right)\right|=t+c r}} \frac{}{\left\langle\partial \rho\left(\tau_{z}\right), \tau_{z}-w\right\rangle}{ }^{n+l} d S(w) .
\end{gathered}
$$

By the proof of lemma 4.7

$$
\left\|L_{4}\right\| \leq \int_{W_{0}} \frac{d V(w)}{\left|\left\langle\partial \rho\left(\tau_{z}\right), \tau_{z}-w\right\rangle\right|^{n+l}} \leq \int_{\Omega} \frac{d V(w)}{\left|\left\langle\partial \rho\left(\tau_{z}\right), \tau_{z}-w\right\rangle\right|^{n+l}} \lesssim 1 .
$$


Applying Stokes' theorem again, now to the domain

$$
\left\{w \in \Omega:\left|\left\langle\partial \rho\left(\tau_{z}\right), \tau_{z}-w\right\rangle\right|<t+c r\right\}
$$

we obtain

$$
\begin{aligned}
L_{5}:=\int_{\substack{w \in \Omega \\
\left|v\left(\tau_{z}, w\right)\right|=t+c r}} & {\overline{\left\langle\partial \rho\left(\tau_{z}\right), \tau_{z}-w\right\rangle}}^{n+l} d S(w) \\
=- & \int_{\substack{w \in \partial \Omega \\
\left|v\left(\tau_{z}, w\right)\right|<t+c r}}{\overline{\left\langle\partial \rho\left(\tau_{z}\right), \tau_{z}-w\right\rangle}}^{n+l} d S(w) \\
+ & \int_{\substack{w \in \Omega \\
\left|v\left(\tau_{z}, w\right)\right|<t+c r}} \bar{\partial}_{w}\left({\overline{\left\langle\partial \rho\left(\tau_{z}\right), \tau_{z}-w\right\rangle}}^{n+l}\right) \wedge d S(w) \\
& +\int_{\substack{w \in \Omega \\
\left|v\left(\tau_{z}, w\right)\right|<t+c r}}{\overline{\left\langle\partial \rho\left(\tau_{z}\right), \tau_{z}-w\right\rangle}}^{n+l} d V(w) .
\end{aligned}
$$

Since $\left|\bar{\partial}_{w}\left({\overline{\left\langle\partial \rho\left(\tau_{z}\right), \tau_{z}-w\right\rangle}}^{n+l}\right) \wedge d S(w)\right| \lesssim\left|\left\langle\partial \rho\left(\tau_{z}\right), \tau_{z}-w\right\rangle\right|^{n+l-1}$ we get

$\left|L_{5}\right| \lesssim \int_{t}^{t+c r}\left(s^{n+l} s^{n-1}+s^{n+l} s^{n}+s^{n+l-1} s^{n}\right) d s \lesssim \int_{t}^{t+c r} s^{2 n+l-1} d s \lesssim r(t+r)^{2 n+l-1}$.

Note that $t \asymp \rho\left(\tau_{z}\right) \asymp \operatorname{Im}\left(\tau_{n}\right)$ and consequently

$$
\begin{aligned}
& \int_{D_{0}} L_{5}(z, \tau)^{2} d \nu_{l}(\tau) \lesssim \int_{D_{0}}\left(\frac{r\left(\operatorname{Re}\left(\tau_{n}\right)+r\right)^{2 n+l-1}}{\left(\operatorname{Re}\left(\tau_{n}\right)+r\right)^{2 n+2 l}}\right)^{2} d \nu_{l}(\tau) \\
& \quad \lesssim \int_{0}^{\infty} \frac{r^{2}}{(t+r)^{2 l+2}} \frac{t^{n} d t}{t^{n-2 l+1}}=r^{2} \int_{0}^{\infty} \frac{t^{2 l-1}}{(t+r)^{2 l+2}} \frac{t^{n} d t}{t^{n-2 l+1}} \lesssim r^{2} \int_{0}^{\infty} \frac{d t}{(r+t)^{3}} \lesssim 1 .
\end{aligned}
$$

Summarizing estimates $(24,25)$, lemma 4.7 and condition $|f(z)| \leq 1, z \in$ 
$\partial \Omega$, we obtain

$$
\begin{aligned}
& \|\langle g, T f\rangle\|^{2} \\
& \leq \int_{D_{0}} d \nu_{l}(\tau)\left(\int_{B\left(w_{0}, r\right)}|g(z)|\left(L_{1}(z, \tau)+|f(z)|\left(L_{2}(z, \tau)+L_{3}(z, \tau)\right)\right) d S(z)\right)^{2} \\
& \quad \lesssim\|g\|_{L^{1}(\partial \Omega)}^{2} \sup _{z \in \partial \Omega} \int_{D_{0}}\left(L_{1}(z, \tau)^{2}+L_{2}(z, \tau)^{2}+L_{3}(\tau, z)^{2}\right) d \nu_{l}(\tau) \\
& \lesssim\|g\|_{L^{1}(\partial \Omega)}^{2} \lesssim\left|B\left(w_{0}, r\right)\right|^{2} .
\end{aligned}
$$

The last estimate implies weak boundedness of operator $T$ and completes the proof of the lemma.

Proof (of the theorem 4.1). Since operators $T_{j}$ with kernels $K_{j}$ verify the conditions of $T 1$-theorem, we have $T_{j} \in \mathscr{L}\left(L^{p}(\partial \Omega), L^{p}\left(\partial \Omega, L^{2}\left(D_{0}, d \nu_{l}\right)\right)\right.$ and

$$
\begin{aligned}
& \|I(g)\|_{L^{p}(\partial \Omega)}^{p} \sum_{j=1}^{N} \int_{\partial \Omega}\left\|T_{j} g(z)\right\|^{p} d S(z) \\
& =\sum_{j=1}^{N} \int_{\partial \Omega} d S(z)\left(\int_{D_{0}}\left|\int_{\partial \Omega} \frac{g(w) \chi_{j}^{1 / 2}(z) J_{j}(z, \tau) d S(w)}{\left\langle\partial \rho\left(\psi_{j}(z, \tau)\right), \psi_{j}(z, \tau)-w\right\rangle^{n+1}}\right|^{2} \frac{d \mu(\tau)}{\operatorname{Re}\left(\tau_{n}\right)^{n-1}}\right)^{p} \\
& \\
& \lesssim\|g\|_{L^{p}(\partial \Omega)}^{p} .
\end{aligned}
$$

Thus by decomposition (15) $\int_{\partial \Omega} I_{l}(g, z)^{p} d \sigma(z) \lesssim \int_{\partial \Omega}|g(z)|^{p} d \sigma(z)$, which proves the theorem.

\section{Boundedness of area-integral $\boldsymbol{I}_{l}(g, z)$ on $\mathrm{BMO}(\partial \Omega)$.}

In this section we prove theorem 4.2. Analogously to theorem 4.1 in is enough to prove that $T_{j} \in \mathcal{L}\left(\operatorname{BMO}(\partial \Omega), \operatorname{BMO}\left(\partial \Omega, L^{2}\left(D_{0}, d \nu_{l}\right)\right)\right)$.

Lemma 5.1. Functions $T_{j}(1), T_{j}^{\prime}(1)$ satisfy Hölder condition with exponent $1 / 2$.

Proof. Similarly to lemma 4.6 we can prove, that

$$
\left\|K_{j}(\xi, w)-K_{j}(z, w)\right\| \lesssim \frac{d(\xi, z)^{1 / 2}}{d(z, w)^{n+1 / 2}}, x, z \in \partial \Omega, w \in \Omega
$$


thus by Jensen's inequality and by expression (20) for $T_{j}(1)$

$$
\begin{aligned}
& \left\|T_{j}(1)(z)-T_{j}(1)(\xi)\right\|=\left\|\int_{\Omega}\left(K_{j}(\xi, w)-K_{j}(z, w)\right) d V(w)\right\| \\
& \lesssim \int_{\Omega}\left\|K_{j}(\xi, w)-K_{j}(z, w)\right\| d V(w) \lesssim \int_{\Omega} \frac{d(\xi, z)^{1 / 2}}{d(z, w)^{n+1 / 2}} \lesssim d(\xi, z)^{1 / 2} .
\end{aligned}
$$

The estimate for $T_{j}^{\prime}$ is obtained analogously.

Lemma 5.2. $T_{j}, T_{j}^{\prime} \in \mathcal{L}\left(\operatorname{BMO}(\partial \Omega), \operatorname{BMO}\left(\partial \Omega, L^{2}\left(D_{0}, d \nu_{l}\right)\right)\right)$.

Proof. Let $b \in \operatorname{BMO}(\partial \Omega)$ and fix a quasiball $B_{\varepsilon}=B\left(z_{0}, \varepsilon\right) \subset \partial \Omega$. We decompose $b$ as a sum $b=b_{1}+b_{2}+b_{3}$, where $b_{1}=b_{B_{C \varepsilon}}, b_{2}=\left(b-b_{1}\right) \chi_{B_{C} \varepsilon\left(z_{0}\right)}$, $\chi_{B_{C \varepsilon}\left(z_{0}\right)}$ is a characteristical function of a quasiball $B_{C \varepsilon}\left(z_{0}\right)$, and $C>0$ is large enough. Here we will Use notation $\left|B_{\varepsilon}\right|=d S\left(B_{\varepsilon}\right)$ (recall that measures $d S$ and $d \sigma$ are equivalent).

By [4] we have $\left|b_{1}\right| \leq\|b\|_{B M O} \log \frac{1}{\varepsilon}$, hence by lemma 5.1

$$
\begin{array}{rl}
\frac{1}{\left|B_{\varepsilon}\right|} \int_{B_{\varepsilon}}\left\|T_{j} b_{1}(z)-\left(T_{j} b_{1}\right)_{B_{\varepsilon}}\right\| d & S(z) \lesssim \frac{\left|b_{1}\right|}{\left|B_{\varepsilon}\right|^{2}} \int_{B_{\varepsilon}} \int_{B_{\varepsilon}}\left\|T_{j} 1(z)-T_{j} 1(\xi)\right\| d S(\xi) d S(z) \\
& \lesssim\left|b_{1}\right| \varepsilon^{1 / 2} \leq \varepsilon^{1 / 2} \log \frac{1}{\varepsilon}\|b\|_{B M O} \lesssim\|b\|_{B M O} .
\end{array}
$$

To estimate $T_{j} b_{2}$ we use the boundedness of

$$
\begin{gathered}
\left(\frac{1}{\left|B_{\varepsilon}\right|} \int_{B_{\varepsilon}}\left\|T_{j} b_{2}-\left(T_{j} b_{2}\right)_{B_{\varepsilon}}\right\| d S\right)^{2} \lesssim\left(\frac{1}{\left|B_{\varepsilon}\right|} \int_{B_{\varepsilon}}\left\|T_{j} b_{2}\right\| d S\right)^{2} \\
\lesssim \frac{1}{\left|B_{\varepsilon}\right|} \int_{\partial \Omega}\left\|T_{j} b_{2}\right\|^{2} d S \lesssim \frac{1}{\left|B_{\varepsilon}\right|} \int_{\partial \Omega}\left|b_{2}\right|^{2} d S \\
=\frac{1}{\left|B_{\varepsilon}\right|} \int_{B_{C \varepsilon}}\left|b(z)-b_{B_{\varepsilon}}\right|^{2} d S(z) \lesssim\|b\|_{B M O}^{2}
\end{gathered}
$$


Finally, estimating $T_{j} b_{3}$ we have

$$
\begin{aligned}
& \frac{1}{\left|B_{\varepsilon}\right|} \int_{B_{\varepsilon}\left(z_{0}\right)}\left\|T_{j} b_{3}-\left(T_{j} b_{3}\right)_{B_{\varepsilon}}\right\| d S \\
& \left.\lesssim \frac{1}{\left|B_{\varepsilon}\right|^{2}} \int_{B_{\varepsilon}} \int_{B_{\varepsilon}}\left\|\int_{\partial \Omega}(K(\xi, w)-K(z, w)) b_{3}(w) d S(w)\right\| d S(z)\right) d S(\xi) \\
& \lesssim \frac{1}{\left|B_{\varepsilon}\right|^{2}} \int_{B_{\varepsilon}} \iint_{B_{\varepsilon}} \frac{d(\xi, z)^{1 / 2}}{d(w, z)^{n+1 / 2}}\left|b_{3}(w)\right| d S(w) d S(z) d S(\xi) \\
& \quad \int_{\partial \Omega \backslash B_{C \varepsilon\left(z_{0}\right)}} \frac{\left|b(w)-b_{C \varepsilon\left(z_{0}\right)}\right|}{d\left(w, z_{0}\right)^{n+1 / 2}} d S(w) \lesssim\|b\|_{B M O},
\end{aligned}
$$

because $d(\zeta, z) \lesssim \varepsilon$ and $d(w, z) \asymp d\left(w, z_{0}\right)$ when $C>0$ is large enough. The proof for $T^{\prime}$ is analogous.

By decomposition (15) this finalizes the proof of the theorem 4.2.

\section{References}

\section{References}

[1] P. Ahern, J. Bruna, Maximal and Area Integral characterization of Hardy-Sobolev Spaces in the unit ball in $\mathbb{C}^{n}$, Rev. Mat. Iberoamericana, Vol. 4, No. 1, 123-153 (1988)

[2] L. A. Aizenberg, A. P. Yuzhakov, Integral Representations and Residues in Complex Analysis [in Russian], Moscow (1979).

[3] E. M. Dyn'kin, Estimates of analytic functions in Jordan domain, Zap. Nauch. Sem. LOMI, 73, 70-90 (1977).

[4] C. Fefferman, E.M. Stein, $H^{p}$ spaces of several variables, Acta mathematica, Vol. 129, No. 1, 137-193 (1972).

[5] L. Grafakos, L. Liu, D. Yang, Vector-valued singular integrals and maximal functions on spaces of homogeneous type, Math. Scand. 104 (2009), 296-310 
[6] S. Krantz, S.Y. Li, Area integral characterizations of functions in Hardy spaces on domains in $\mathbb{C}^{n}$, Complex Variables, Vol. 32, No. 4, 373-399 (1997).

[7] T. Hytönen, L. Weis, A T1 theorem for integral transformations with operator-valued kernel, J. for Pure and Applied Math., Vol. 2006, No. 599, 155-200 (2006).

[8] L. Lanzani, E. M. Stein, Cauchy-type integrals in several complex variables, Bull. Math. Sci., Vol. 3, No.2, 241-285 (2013).

[9] L. Lanzani, E. M. Stein, The Cauchy Integral in $\mathbb{C}^{n}$ for domains with minimal smoothness, Adv. Math. 264, 776-830 (2014).

[10] J. Leray, Le calcul différentiel et intégral sur une variátá analytique complexe. (Problème de Cauchy. III.) Bull. Soc. Math. Fr. 87, 81-180 (1959).

[11] A. Nagel, E.M. Stein, S. Wainger, Boundary behaviour of functions holomorphic in domains of finite type. Proc. Nat. Accad. Sci. US, Vol. 78, No. 11, part 1, 6596-6599, (1981).

[12] R. M. Range, Holomorphic functions and integral representations in several complex variables, Springer Verlag (1986).

[13] A. S. Rotkevich, The Cauchy-Leray-Fantappi'e integral in linearly convex domains, Zap. Nauch. Sem. POMI 401, 172-188 (2012).

[14] A. S. Rotkevich, Constructive description of the Besov classes in convex domains in $\mathbb{C}^{n}$, Zap. Nauch. Sem. POMI 401, 136-174 (2013).

[15] G. Sandrine, Complex tangential characterizations of Hardy-Sobolev Spaces of holomorphic functions, Rev. Mat. Iberoamericana, Vol. 9, 201-255 (1993).

[16] N. A. Shirokov, Jackson-Bernstein theorem in strictly pseudoconvex domains in $\mathbb{C}^{n}$, Constr. Approx., Vol. 5, No. 1, 455-461 (1989). 
[17] N. A. Shirokov, A direct theorem for strictly convex domains in $\mathbb{C}^{n}$, Zap. Nauch. Sem. POMI 206, 152-175 (1993).

[18] E.M. Stein, On the functions of Littlewood-Paley, Lusin, Marcinkiewicz, Trans. Amer. Math. Soc., Vol. 88, 430-466 (1958). 